\title{
The glycosylated extracellular domain of MUC1 protects against SARS-CoV-2 infection at the respiratory surface
}

Maitrayee Chatterjee ${ }^{1}$, Liane Z.X. Huang ${ }^{1}$, Chunyan Wang ${ }^{1}$, Anna Z. Mykytyn ${ }^{3}$, Bart Westendorp ${ }^{2}$, Richard W. Wubbolts ${ }^{2}$, Berend-Jan Bosch ${ }^{1}$, Bart L. Haagmans ${ }^{3}$, Jos P.M. van Putten ${ }^{1}$ and Karin Strijbis ${ }^{1 *}$

${ }^{1}$ Department of Biomolecular Health Sciences, Division Infectious Diseases and Immunology, Faculty of Veterinary Medicine, Utrecht University, The Netherlands

2 Department of Biomolecular Health Sciences, Division Cell Biology, Metabolism and Cancer, Faculty of Veterinary Medicine, Utrecht University, The Netherlands

${ }^{3}$ Viroscience Department, Erasmus Medical Center, Rotterdam, The Netherlands

*Corresponding author. Email: K.Strijbis@uu.nl 


\begin{abstract}
Mucins play an essential role in protecting the respiratory tract against microbial infections. The heavily $O$-glycosylated gel-forming mucins MUC5AC and MUC5B eliminate pathogens by mucociliary clearance while transmembrane mucins MUC1, MUC4, and MUC16 restrict microbial invasion at the apical surface of the epithelium. In this study, we determined the impact of host mucins and mucin glycans on SARS-CoV-2 spike-mediated epithelial entry. Human lung epithelial Calu-3 cells have endogenous expression of the SARS-CoV-2 entry receptor ACE2 and express high levels of glycosylated MUC1 on the surface but not MUC4 and MUC16. Removal of the MUC1 extracellular domain (ED) using the $O$-glycan-specific mucinase StcE greatly enhanced spike binding and viral infection. By contrast, removal of mucin glycans sialic acid and fucose did not impact viral invasion. This study implicates the glycosylated ED of MUC1 as an important component of the host defense that restricts the severity of SARS-CoV-2 infection.
\end{abstract}

Keywords: Mucins, transmembrane mucin, cell-bound mucin, MUC1, O-linked glycans, sialic acid, fucose, SARS-CoV-2, ACE2, spike, mucin-microbe interactions 


\section{Introduction}

During entry through the respiratory system, pathogens encounter a defensive mucus system that protects the underlying epithelium. The major components of mucus are heavily $O$-glycosylated mucin glycoproteins. Soluble mucins are secreted by Goblet cells and provide mucus threads for mucociliary clearance (MCC) of particles and pathogens. Transmembrane mucins are expressed on the apical membrane and cilia and prevent access to epithelial surface receptors ${ }^{1}$. SARS-CoV-2, the coronavirus that is responsible for the COVID-19 pandemic, is an enveloped, single-stranded, positive-sense RNA virus that belong to the $\beta$ coronavirus genus within the Coronaviridae family ${ }^{2,3}$. SARS-CoV-2 preferentially utilizes receptorangiotensin-converting enzyme 2 (ACE2) as entry receptor by interaction with its envelope-anchored spike (S) protein ${ }^{4}$. In addition to ACE2, SARS-CoV-2 entry requires proteolytic cleavage of the spike protein that can be mediated by the transmembrane serine protease 2 (TMPRSS2) $)^{5}$. Human coronaviruses have also been described to depend on sialic acids linked to glycoproteins or gangliosides as primary attachment sites in the respiratory tract $^{6}$. Glycosylated mucins can be decorated with sialic acids, but the role of mucin glycans and specific mucins during SARS-CoV-2 entry remains to be established.

The major mucins of the respiratory system are soluble mucins MUC5AC and MUC5B, and transmembrane (TM) mucins MUC1, MUC4, and MUC16 ${ }^{1}$. MUC1 and MUC4 are expressed in the upper and lower airway epithelium, whereas MUC16 expression is restricted to the lower airways ${ }^{7}$. The high molecular weight mucin glycoproteins contain domains with extensive $O$-glycan structures that often terminate with charged sialic acids or hydrophobic fucoses that impact their interaction with microbes ${ }^{8}$. The expression and glycosylation profiles of mucins are directly influenced by colonization and invasion by bacteria and viruses and are altered during inflammation of the respiratory tract ${ }^{9}$. Transmembrane mucins form filamentous structures that extend above the apical surface of the epithelium and these mucins consist of a heavily $\mathrm{O}$-glycosylated $\mathrm{N}$-terminal extracellular domain (ED), a single transmembrane domain, and a C-terminal cytoplasmic domain (CT) with signaling capacity. In the lung, MUC1 primarily expresses around microvilli and protrudes at least 100 $\mathrm{nm}$ from the cell surface whereas MUC4 ( $300 \mathrm{~nm}$ in size), and the even larger MUC16 are expressed on the surface of cilia ${ }^{10}$. Together, the TM mucins form a barrier that restricts access to the underlying epithelium, act as releasable decoy receptors, and sterically hinder the binding of pathogens to underlying cellular receptors ${ }^{11}$. MUC1 has been most extensively studied and implicated in defense against respiratory infections with Pseudomonas aeruginos $a^{12}$ and respiratory syncytial virus ${ }^{13}$ and Influenza A virus infection ${ }^{14}$. In this present study, we determined the impact of transmembrane mucins on spike-mediated entry of SARS-CoV-2 in human respiratory cells. MUC1 is the dominant 
transmembrane mucins and removal of the extracellular domain greatly enhances spike binding and viral infection. This study points to critical importance of MUC1 in limiting SARS-CoV-2 infection.

\section{Results}

\section{ACE2-positive respiratory cells express high levels of transmembrane mucin MUC1}

The Human Cell Atlas consortium respiratory single cell RNA-seq dataset allows analysis of gene expression in the nasal cavity and proximal, intermediate, and distal respiratory tract ${ }^{15}$. We analysed this dataset to determine the expression of ACE2 and mucins in different respiratory cell types present in the upper and lower respiratory mucosa. ACE2-positive cells included secretory, basal, suprabasal and multiciliated cells and the majority of secretory and multiciliated cells expressed MUC1 (Fig 1A). Next, we determined which mucins are most highly expressed in ACE2-positive cells in the respiratory tract. The most highly expressed TM mucin in ACE2-positive cells was MUC1 followed by TM mucins MUC4 and MUC16 and soluble mucins MUC5AC and MUC5B (Fig 1B). This analysis suggests that the TM mucin MUC1 is the dominant mucin in ACE2-positive respiratory cells in healthy individuals.

Human respiratory Calu-3 cells have high expression of ACE2 and TMPRSS2 and are highly susceptible to SARS-CoV-2 spike-mediated entry ${ }^{16,17,5}$. We first determined the expression of different mucins and their glycans on Calu-3 cells by immunofluorescence confocal microscopy. Multiple Z-stack images were collected which showed expression of MUC1, MUC4, and MUC5AC and only very limited expression of MUC16 (Fig 1C, S1A respectively). To distinguish which mucins are expressed on the extracellular surface, we performed immunofluorescence staining without permeabilization of the Calu-3 cells. Using this method, MUC1 was clearly detectable on the surface whereas MUC4 and MUC5AC could not be stained indicating an intracellular localization (Fig 1D). Next, we determined the expression of the terminal mucin glycans sialic acid and fucose on Calu-3 cells. Immunofluorescence with SNA, MALII, and UEA-I lectin showed the presence of $\alpha-2,6$ sialic acid, $\alpha-2,3$ sialic acid, and fucose on Calu-3 cells, respectively (Fig 1E). The $\alpha-2,6$ sialic acid and $\alpha-2,3$ sialic acid signals (SNA and MALII) were more prominently detected at the edge of the cell island compared to the fucose signal (UEA1) and some colocalization with MUC1 could be observed. These results demonstrate that Calu-3 cells endogenously express MUC1 on their surface and have abundant expression of sialic acids and fucose.

\section{StcE specifically cleaves the MUC1 ED and does not affect ACE2 expression}

The StcE mucinase recognizes an $O$-glycosylated serine-threonine motif that is abundant in mucins and is virtually absent in non-mucin proteins ${ }^{18}$. We previously applied this bacterial mucinase and its inactive point mutant E447D to remove the MUC1 ED ${ }^{19}$. To investigate the effect of StcE on endogenous MUC1 expressed by Calu-3 cells, confocal microscopy was performed on non-treated, StcE-treated and E447D-treated Calu-3 cells and stained with $\alpha$-MUC1-ED antibody 214D4, $\alpha$-MUC1- 
SEA antibody 232A1, and $\alpha-M U C 1-C T$ antibody CT2. StcE treatment efficiently removed the glycosylated part of the MUC1 extracellular domain as indicated by a complete loss of $\alpha$-MUC1-ED 214D4 staining after incubation with the enzyme (Fig 2A). The MUC1 SEA domain and CT are predicted not to be digested by StcE and indeed both domains remained detectable after enzyme treatment (Fig $2 B, 2 C)$. We next investigated the effect of StcE, E447D, neuraminidase, and fucosidase treatment on MUC1 by Western blot. Calu-3 cells were incubated with the enzymes for $3 \mathrm{~h}$ and then subjected to Western blot analysis with the $\alpha$-MUC1-ED antibody 214D4 and $\alpha$-MUC1-CT antibody CT2. After incubation with StcE, the glycosylated part of the extracellular domain of MUC1 (about $450 \mathrm{kDa}$ ) was no longer detectable. The high molecular weight MUC1 band was not affected by treatment with the inactive enzyme E447D or fucosidase. After neuraminidase treatment on the other hand, we did observe a reduction of the MUC1 signal which could be caused by a change in antibody recognition or altered protein transfer to the membrane (Fig 2D). The observed banding pattern for the MUC1 cytoplasmic tail was not affected by the enzymatic treatments (Fig 2E). Furthermore, we wanted to determine the effect of enzymatic treatment on ACE2 stability because the ACE2 receptor itself is glycosylated ${ }^{20}$. No change in expression of the full-length glycosylated ACE2 (nearly $140 \mathrm{kDa}$ ) could be observed after treatment with StcE, E447D, neuraminidase, or fucosidase. Interestingly, the soluble form of ACE2 (around $70 \mathrm{kDa}$ ) was more prominently detectable after fucosidase treatment (Fig 2F). These results demonstrate that StcE cleaves the glycosylated part of the MUC1 ED without affecting ACE2 expression in Calu-3 cells.

\section{Enzymatic removal of the extracellular domain of MUC1 enhances SARS-CoV-2 entry}

MUC1 is heavily decorated with $O$-glycans. To investigate whether these individual glycans or glycosylated ED of MUC1 form a barrier against SARS-CoV-2, we removed 0 -glycan sugars using neuraminidase, fucosidase and StcE was used to cleave the MUC1 ED. Surface $\alpha 2,3-, \alpha 2,6-$, and $\alpha 2,8-$ linked sialic acids were removed by incubation with neuraminidase and fucose by fucosidase treatment. Neuraminidase treatment for $3 \mathrm{~h}$ removed the majority of surface-exposed $\alpha 2,3$ - linked sialic acids as detected by MAL-II staining, and $\alpha 2,6$-linked sialic acids detected by SNA staining (S1B). Similarly, fucosidase treatment for $3 \mathrm{~h}$ cleaved surface-exposed fucose which was detected by UEA1 staining (S1C). StcE treatment effectively removed the MUC1 glycosylated ED from the cellular surface as describe above (Fig 2). After enzymatic treatment of the Calu-3 cells, a SARS-CoV-2 pseudotyped virus carrying the spike protein and encoding a GFP reporter (SARS2-S pseudotyped VSV-GFP) was added in the absence or presence of an anti-spike monoclonal antibody and incubated for 24 hours. StcE treatment greatly enhanced the number of SARS2-S pseudotyped VSV-GFP positive cells, while E447D-treated cells did not show enhanced viral entry. No obvious change in viral infection could be 
observed after neuraminidase or fucosidase treatment (Fig 3A). In all experimental conditions, viral infection was completely blocked in the presence of the monoclonal antibody against the SARS-CoV2 spike protein demonstrating that we are looking at spike-mediated entry in our experimental setup. The GFP signal was quantified using ImageJ showing a significant 5.4-fold increase in Calu-3 virus infection after StcE treatment and no significant difference after neuraminidase and fucosidase treatment (Fig 3B).

In an independent set of experiments with a luciferase pseudovirus (SARS2-S pseudotyped VSV-Luc), we also observed a 4-fold increase in viral infection after StcE treatment (Fig 3C). As an additional control, we performed the infection with an VSV-G pseudotyped VSV-Luc that lacks the spike protein. We observed enhanced entry of VSV-G pseudotyped VSV-Luc into Calu-3 after StcE and neuraminidase treatment whereas fucosidase treatment had an opposite effect. As expected, the infection could not be blocked with the mAb against spike (Fig 3D).

Next, we investigated the effect of mucin removal on infection with the authentic SARS-CoV-2 virus. Calu-3 cells were treated with the enzymes and incubated with SARS-CoV-2 virus in the absence or presence of an anti-spike monoclonal antibody for 8 hours to study initial entry. In line with our pseudovirus experiments, we observed a significant increase in the number of infected cells when cells were treated with StcE mucinase in comparison to control. Again, neuraminidase and fucosidase treatment did not significantly impact viral infection albeit we observed a trend towards increased infection after neuraminidase treatment (Fig 3E).

To understand the spatial relationship between the ACE2 receptor and the MUC1 transmembrane mucin, we performed confocal microscopy on Calu-3 cells stained for ACE2 receptor and the MUC1 extracellular domain. ACE2 signal was observed as scattered puncta across the monolayer and a few bright dome-like structures (Fig 3F, G). Both staining types were also visible in StcE and E447D-treated cells (Fig S2A-D). StcE treatment resulted in a complete loss of MUC1-ED staining but did not visibly alter expression levels or subcellular localization of ACE2 (Fig S2A, B). Interestingly, in the dome-like structures we observed a clear layer of MUC1 above the ACE2 signal, possibly indicative of a protective function of the MUC1 glycosylated ED (Fig 3G, side view). Confocal microscopy on Calu-3 cells infected with SARS2-S pseudotyped VSV-GFP confirmed that StcE treatment increased the number of infected cells (Fig $3 \mathrm{H}$ ). Together these data demonstrate that removal of the MUC1 glycosylated ED results in increased SARS-CoV-2 infection of lung epithelial cells without affecting ACE2 expression. No effect on viral entry was observed after removing individual glycans sialic acid and fucose.

Negatively charged molecules such as sialic acid or heparan sulphate (HS) on the cellular surface or extracellular matrix proteoglycans have been described to facilitate viral entry ${ }^{21,22}$. Therefore, we investigated if heparanase treatment to remove HS or neuraminidase treatment to remove sialic acids 
impacted viral invasion after removal of the MUC1 glycosylated domain with StcE. Calu-3 cells were first treated with StcE, followed by treatment with heparanase or neuraminidase and subsequent viral infection with SARS2-S pseudotyped VSV-Luc. Confocal microscopy confirmed the removal of HS and $\alpha-2,6$ sialic acid from the surface of Calu-3 cells after heparanase and neuraminidase treatment, respectively (Fig S2A, B). Quantification of viral infection showed that the combination treatments did not significantly impact viral invasion compared to StcE only condition (Fig S3C). A small reduction of viral infection was observed when the cells were treated with only heparanase in comparison to the control cells without treatment. All infections in this experiment could be blocked by the mAb demonstrating spike-mediated infection. This result suggests that SARS-CoV-2 entry does not depend on these negatively charged molecules on the cell surface of Calu-3 cells.

\section{Removal of the MUC1 ED enhances spike and virus attachment to Calu-3 cells}

In a final set of experiments, we investigated if removal of the MUC1 ED directly affected spike and virus attachment to the cellular surface. Calu-3 cells were treated with StcE followed by incubation with purified Fc tagged spike protein (SARS2-S1B-FC) or SARS2-S pseudotyped VSV-GFP for $1 \mathrm{~h}$ at $4^{\circ} \mathrm{C}$ to monitor attachment and prevent entry. The spike protein was stained without first permeabilizing the prevent detection of intracellular spike. In untreated and E447D-treated cells, spike binding was observed in patches along the edge of the cell island while cells treated with StcE showed extensive staining (Fig 4A). Quantification of the fluorescent spike signal on the edges of the cell islands using ImageJ confirmed a significant increase in StcE-treated cells raw as determined by integrated density/length (sum of all pixels/ $\mu \mathrm{m}$ ) (Fig 4B). In line with these findings, also more SARS2-S pseudotyped VSV-GFP virus was able to attach to Calu-3 cells after StcE treatment (Fig 4C). Together these results show that enzymatic removal of the MUC1 extracellular domain allows more virus attachment to cells and thus increases infectivity.

\section{Discussion}

The mucosal barrier is the body's first line of defense and offers protection from infection by pathogens. However, mucins can also serve as attachment sites for bacterial and viral pathogens. The findings presented in this study demonstrate that the transmembrane mucin MUC1 plays a substantial protective role during SARS-CoV-2 infection at the respiratory surface. We show that MUC1 is the dominant mucin in ACE2-positive cells in the respiratory epithelium and that human lung epithelial Calu-3 cells expressed high levels of MUC1, while TM mucins MUC4 and MUC16 and secreted mucin MUC5AC were barely detectable (Fig 1, 2). In various SARS-CoV-2 infection studies, we demonstrate that the large MUC1 glycoprotein forms a protective layer that prevents access of the virus to the underlying ACE2 receptor (Fig 3). Our experiments indicate that enzymatic removal of the extracellular 
domain of MUC1 enhances viral infection of lung epithelial cells (Fig 3). In the absence of the MUC1 glycosylated domain, increased binding of purified spike protein and virus to the cellular surface was observed (Fig 4).

There is growing evidence for the protective role of soluble and transmembrane mucins during SARSCoV-2 infection. In the course of COVID-19, elevated levels of gel-forming MUC5AC and shed MUC1 can be detected in sputum aspirated from the trachea of patients ${ }^{23}$. The MUC5B genetic variant rs35705950 is associated with higher expression of the soluble mucin MUC5B, is underrepresented in COVID-19 patients compared to healthy individuals, suggesting a protective role for MUC5B ${ }^{24}$. It cannot be excluded that decreased mucus production and weakened MCC contribute to the higher susceptibility of SARS-CoV-2 in aged individuals ${ }^{25}$. Our findings on the role of protective endogenous MUC1 are supported by a recent genome-scale CRISPR loss- and gain-of-function study for SARS-CoV2 entry in human lung epithelial cells overexpressing TMs such as MUC1, MUC4 or MUC21 ${ }^{26}$. Gain-offunction (GOF) cell lines overexpressing MUC1, MUC4 or MUC21 showed reduced infection by SARSCoV-2 Spike-pseudotyped VSV compared to cells with non-targeting guide (NTG). The study showed, enzymatic removal of overexpressed MUC4 also resulted in increased viral entry showing that different TM mucins might have a shared protective function during SARS-CoV-2 infection. MUC4 was previously implicated in protection against SARS-CoV in a study with female mice in which MUC4 knockout mice had enhanced inflammatory cytokine responses and poor prognosis ${ }^{27}$. Our single-cell analysis showed that in MUC1 is the dominant mucin in ACE2-positive cell in the respiratory tract in healthy people. Single cell sequencing data of COVID19 patients demonstrated that MUC1, MUC4, MUC13 and MUC21 are all highly upregulated in patients with active disease ${ }^{26}$. Establishing the function of the different mucins during infection is an important future challenge.

The studies that are currently available underscore the importance of extracellular domain of transmembrane mucins during viral entry. Our confocal microscopy analysis demonstrated that the MUC1 glycosylated extracellular domain is covering the ACE2-positive surface. Enzymatic removal of the MUC1 glycosylated domain did not affect the underlying SEA domain or cytoplasmic tail and ACE2 expression remained detectable. As was previously hypothesized, it is possible that MUC1 and ACE2 interact and/or are in the same protein complex on the respiratory surface ${ }^{28}$. Our data indicate that steric hindrance of the large glycosylated extracellular domain of MUC1 prevents the virus from reaching the ACE2 receptor (Fig 5). This is in line with a recent study that used mucin mimetics glycopolymers that were capable of shielding surface receptors ${ }^{29}$. In a previous study, we have shown that MUC1 ED alters the cell membrane to tubulated morphology and reduce $\beta 1$-integrin-mediated 
bacterial invasion ${ }^{19}$. In the present study, we have not observed any influence of MUC1 ED on membrane architecture.

Different studies describe that for viral entry SARS-CoV-2 benefits from negatively charged residues like sialic acid-containing glycans or membrane glycosaminoglycans such as heparan sulfate proteoglycans on the cell surface $\mathrm{e}^{30,31,32,33}$. In contrast with this findings, neuraminidase treatment of Calu-3 cells modestly increased SARS-CoV-2 infection ${ }^{34}$. In our live virus experiments we observed a modest increase in SARS-CoV-2 efficiency after neuraminidase treatment and no effect of fucosidase treatment. We addressed if the negatively charged sialic acids or heparan sulfates were important for viral entry after removal of the glycosylated mucin domain. Consecutive treatment with StcE and neuraminidase or heparinase was performed but did not result in a difference in viral entry. Differences in viral dependence on negatively charged surface molecules maybe be explained by levels of ACE2 and TMPRSS2 protease expression and accessibility of the receptor for the viral spike protein in different cell systems. Therefore, our findings reveal that during infection of human respiratory Calu-3 cells the MUC1 extracellular domain rather than individual mucin glycans prevents the binding of SARS-CoV-2 to the underlying receptor.

In addition to the role of transmembrane mucins in preventing viral entry into epithelial cells, mucins can have a detrimental effect during the later stages of COVID-19. Overproduction and excess accumulation of gel-forming mucins in the lungs of COVID-19 patients can lead to airway obstruction and eventually cause life-threatening ARDS ${ }^{35,36,37}$. Several studies are focusing on the reduction of mucin expression as a therapeutic strategy $38,39,40$. Compound R406, the active metabolite of FDAApproved Fostamatinib that inhibits MUC1 expression is now in clinical trials for hospitalized patients with advanced COVID-1941. Our results on the important function of MUC1 in preventing SARS-CoV-2 infection could point towards a strategy to promote MUC1 expression, boost mucosal defense mechanisms and prevent SARS-CoV-2 infection in the first place.

\section{Methods}

\section{Single cell analysis}

Normalized counts and metadata from previously published single cell RNA-sequencing data of healthy human airway epithelium ${ }^{15}$ were downloaded from https://www.genomique.eu/cellbrowser/HCA/. Dimensionality reduction was done using the Seurat Package $^{42}$ in Rstudio (version 1.2.5019), starting with a principle component analysis. After visual inspection of the principal components using and elbow plot, the first twenty components were used 
for graph-based clustering analysis. Clusters of cells were then visualized as diffusion maps (uMAPs). To determine gene expression in ACE2- and TMPRSS2-positive versus negative cells we created two additional metadata slots, in which normalized transcript counts of these genes above 0 were considered positive. Then, cell type assignment and normalized expression of a panel of genes of interest was determined by sub-setting single or double-positive epithelial cells.

\section{Cell culture}

Calu-3 cells (ATCC Catalog \# HTB-55), HEK-293T (ATCC Catalog \# CRL-3216) and BHK-21 cells (ATCC Catalog \# CCL-10) cells were routinely grown in $25 \mathrm{~cm}^{2}$ flasks in Dulbecco's modified Eagle's medium (DMEM) containing $10 \%$ fetal calf serum (FCS) at $37^{\circ} \mathrm{C}$ in $5 \% \mathrm{CO}_{2}$.

\section{Production of SARS-CoV-2 pseudotyped virus and virus neutralization assay}

The pseudotyped vesicular stomatitis virus (VSV) was produced by using the protocol of Whitt ${ }^{43}$. The detailed protocol of the production of pseudotyped VSV, SARS2-Spike pseudotyped VSV virus and virus neutralization assay is described in the supplementary methods. The optimal working concentration of SARS2-Spike pseudotyped VSV particles (SARS2-S pseudotyped VSV-GFP and SARS2-S pseudotyped VSV-Luc) was determined by viral titration assay on Calu-3 cells.

\section{Production of authentic SARS-CoV-2 virus stock}

SARS-CoV-2 (isolate BetaCoV/Munich/BavPat1/2020; European Virus Archive Global \#026V-03883; kindly provided by Dr. C. Drosten) was propagated on Calu-3 cells in OptiMEM I (1X) + GlutaMAX (Gibco), supplemented with penicillin $(100 \mathrm{IU} / \mathrm{mL})$ and streptomycin $(100 \mathrm{IU} / \mathrm{mL})$ at $37^{\circ} \mathrm{C}$ in a humidified $\mathrm{CO}_{2}$ incubator. Stocks were produced as described previously ${ }^{44}$. A detailed description of virus production can be found in the supplementary methods.

\section{Enzyme treatment of Calu-3 cells}

StcE and StcE-E447D were expressed and purified as described previously ${ }^{19}$. For mucinase treatment, Calu-3 cells were treated with $2.5 \mathrm{ug} / \mathrm{ml}$ of StcE or its inactive mutant E447D in 10\% FCS media for $3 \mathrm{~h}$ at $37^{\circ} \mathrm{C}$ and washed with DPBS. Desialylation of Calu-3 cells was achieved by incubating cells grown in a 96 well plate or 24-well plate or 6 well plate with $100 \mathrm{U} / \mathrm{mL} \alpha 2-3,6,8,9$ neuraminidase A (P0722L, NEB) in $10 \%$ FCS media for $3 \mathrm{~h}$ at $37^{\circ} \mathrm{C}$. For fucosidase treatment of Calu-3 cells, $0.4 \mathrm{U} / \mathrm{ml}$ of $\alpha-(1-$ 2,3,4,6)-L-Fucosidase (E-FUCHS; Megazyme) was added to the cells and incubated for $3 \mathrm{~h}$ at $37^{\circ} \mathrm{C}$. To remove heparan sulfate (HS), $0.1 \mathrm{U} / \mathrm{ml}$ heparinase III (H8891-5UN, Sigma) was applied as described 
for the other enzymes. After enzyme treatment, cells were washed thrice with DPBS and used for subsequent experiments.

\section{SARS-CoV-2 infection assays}

For infection experiments, Calu-3 cells were grown in 96-well plates and allowed to reach around $90 \%$ confluency. Then, cells were treated with enzymes for $3 \mathrm{~h}$ at $37^{\circ} \mathrm{C}$ and $5 \% \mathrm{CO} 2$, before they were inoculated with SARS2-S pseudotyped VSV-Luc or SARS2-S pseudotyped VSV-GFP. At $20 \mathrm{~h}$ postinfection, culture supernatants were aspirated, washed with DPBS, and cells were lysed by overnight incubation with Renilla luciferase assay lysis buffer (Promega) at $-80^{\circ} \mathrm{C}$. The next day, cell lysates were thawed, thoroughly resuspended, and transferred to white, opaque-walled 96-well plates and relative luminescence unit (RLU) was measured. Renilla luciferase activity was determined using the Luciferase Assay Systems (Promega) according to the manufacturer's instructions. Raw luminescence values were recorded as counts per 5 seconds by Berthold Centro LB 942 plate luminometer. For SARS2-S pseudotyped VSV-GFP mediated infection, GFP positive signal captured using an EVOS microscope (Thermo Scientific) at $4 \mathrm{X}$ magnification and quantified using EVOS software. For infection experiments with the authentic SARS-CoV-2 virus, Calu-3 cells were prepared as described above and inoculated with approximately $200 \mathrm{pfu}$ of SARS-CoV-2. At $8 \mathrm{~h}$ post-infection, cells were washed in PBS, fixed in formalin, permeabilized in 70\% ethanol and washed in PBS again. Immunofluorescent stainings were performed as described for SARS-CoV-2 stock production and scanned plates were analyzed using ImageQuant TL software. All work with infectious SARS-CoV-2 was performed in a Class II Biosafety Cabinet under BSL-3 conditions at Erasmus Medical Center.

\section{Confocal Microscopy}

Cells were grown on coverslips up to $80 \%$ confluency were analyzed by immunofluorescent staining. Cells were washed twice with DPBS and fixed with 4\% paraformaldehyde in PBS (Affymetrix) for 20 min at room temperature and fixation was stopped with $50 \mathrm{mM} \mathrm{NH}_{4} \mathrm{Cl}$ in PBS for $10 \mathrm{~min}$. The staining procedure and antibody details are described in the supplementary methods.

\section{Western blotting}

Calu-3 cells were grown in 6-well plates for 7 days before enzyme treatment. Enzyme-treated cells were washed thrice with cold DPBS and collected with a scraper. The cell suspension was centrifuged at 5,000 rpm for $5 \mathrm{~min}$ at $4^{\circ} \mathrm{C}$. Cell pellets were resuspended with $100 \mu \mathrm{l} \%$ SDS in presence of a Halt protease inhibitor cocktail and 0.5 M EDTA solution (Thermo Fisher) and cells lysed mechanically by scratching. Protein concentrations were measured using a BCA protein assay kit (23235\#, Pierce 
Company). For detection of the MUC1 ED, 5\% mucin gels and a boric acid-Tris system were used as described previously ${ }^{45}$. $\alpha$-MUC1-ED antibody 214D4 was used to detect MUC1 at a dilution of 1:1,000 in TSMT buffer. For detection of the CT of MUC1, 12\% SDS-PAGE gel and $\alpha$-MUC1-CT antibody CT2 was used. For ACE2 detection, 10\% SDS-PAGE gel and anti-ACE2 antibody (1:1,000, HPA000288, SigmaAldrich) was used. Actin was detected using $\alpha$-actin antibody (1:5,000; bs-0061R, Bioss). Secondary antibodies used were $\alpha$-mouse IgG secondary antibody (1:10,000; A2304, Sigma), $\alpha$-Armenian hamster IgG (1:10,000; GTX25745, Genetex) and $\alpha$-rabbit IgG (1:10,000; A4914, Sigma). Blots were developed with the Clarity Western ECL kit (Bio-Rad) and imaged in a Gel-Doc system (Bio-Rad).

\section{Statistical Analysis}

For all experiments, at least three independent biological replicates were performed. Values are expressed as the mean \pm SEM of three independent experiments performed in triplicate. Repeated measures one way-ANOVA with Dunnett's post-hoc was applied to test for statistical significance. P values of 0.05 or lower were considered statistically significant. Symbols used are $p>0.05$ (ns, not significant), $\mathrm{p}<0.05(*), p<0.01(* *), p<0.001(* * *), p<0.0001(* * *)$. The GraphPad Prism 9 software package was used for all statistical analyses.

\section{References}

1. Lillehoj, E. P., Kato, K., Lu, W. \& Kim, K. C. Cellular and Molecular Biology of Airway Mucins. in Int Rev Cell Mol Biol. 303, 139-202 (2013).

2. da Costa, V. G., Moreli, M. L. \& Saivish, M. V. The emergence of SARS, MERS and novel SARS-2 coronaviruses in the 21st century. Arch. Virol. 165, 1517-1526 (2020).

3. Hartenian, E. et al. The molecular virology of coronaviruses. J. Biol. Chem. 295, 12910-12934 (2020).

4. Li, F. Receptor Recognition Mechanisms of Coronaviruses: a Decade of Structural Studies. J. Virol. 89, 1954-1964 (2015).

5. Hoffmann, M. et al. SARS-CoV-2 Cell Entry Depends on ACE2 and TMPRSS2 and Is Blocked by a Clinically Proven Protease Inhibitor. Cell 181, 271-280 (2020).

6. Fantini, J., Di Scala, C., Chahinian, H. \& Yahi, N. Structural and molecular modelling studies reveal a new mechanism of action of chloroquine and hydroxychloroquine against SARS-CoV2 infection. Int. J. Antimicrob. Agents 55, 105960 (2020).

7. Ridley, C. \& Thornton, D. J. Mucins: The frontline defence of the lung. Biochem. Soc. Trans. 46, 
1099-1106 (2018).

8. Bennett, E. P. et al. Control of mucin-type O-glycosylation: A classification of the polypeptide GalNAc-transferase gene family. Glycobiology 22, 736-756 (2012).

9. Chatterjee, M., van Putten, J. P. M. \& Strijbis, K. Defensive properties of mucin glycoproteins during respiratory infections-relevance for Sars-CoV-2. MBio 11, e02374-20 (2020).

10. Hattrup, C. L. \& Gendler, S. J. Structure and Function of the Cell Surface (Tethered) Mucins. Annu. Rev. Physiol. 70, 431-457 (2008).

11. Van Putten, J. P. M. \& Strijbis, K. Transmembrane Mucins: Signaling Receptors at the Intersection of Inflammation and Cancer. J. Innate Immun. 9, 281-299 (2017).

12. Ueno, K. et al. MUC1 mucin is a negative regulator of toll-like receptor signaling. Am. J. Respir. Cell Mol. Biol. 38, 263-268 (2008).

13. Li, Y., Dinwiddie, D. L., Harrod, K. S., Jiang, Y. \& Kim, K. C. Anti-inflammatory effect of MUC1 during respiratory syncytial virus infection of lung epithelial cells in vitro. Am. J. Physiol. - Lung Cell. Mol. Physiol. 298, 558-563 (2010).

14. McAuley, J. L. et al. The cell surface mucin MUC1 limits the severity of influenza A virus infection. Mucosal Immunol. 10, 1581-1593 (2017).

15. Deprez, M. et al. A single-cell atlas of the human healthy airways. Am J Respir Crit Care Med. 202, 1636-1645 (2020).

16. Ou, X. et al. Characterization of spike glycoprotein of SARS-CoV-2 on virus entry and its immune cross-reactivity with SARS-CoV. Nat. Commun. 11, (2020).

17. Cagno, V. SARS-CoV-2 cellular tropism. The Lancet Microbe 1, e2-e3 (2020).

18. Shon, D. J. et al. An enzymatic toolkit for selective proteolysis, detection, and visualization of mucin-domain glycoproteins. Proc. Natl. Acad. Sci. U. S. A. 117, 21299-21307 (2020).

19. Li, X., Wubbolts, R. W., Bleumink-Pluym, N. M. C., van Putten, J. P. M. \& Strijbis, K. The transmembrane mucin muc1 facilitates b1-integrin-mediated bacterial invasion. MBio 12, (2021).

20. Allen, J. D., Watanabe, Y., Chawla, H., Newby, M. L. \& Crispin, M. Subtle Influence of ACE2 Glycan Processing on SARS-CoV-2 Recognition. J. Mol. Biol. 433, 166762 (2021).

21. Milewska, A. et al. Human Coronavirus NL63 Utilizes Heparan Sulfate Proteoglycans for 
Attachment to Target Cells. J. Virol. 88, 13221-13230 (2014).

22. Lindahl, U. \& Li, J. ping. Chapter 3 Interactions Between Heparan Sulfate and Proteins-Design and Functional Implications. International Review of Cell and Molecular Biology vol. 276 (Elsevier Inc., 2009).

23. Lu, W. et al. Elevated MUC1 and MUC5AC mucin protein levels in airway mucus of critical ill COVID-19 patients. J. Med. Virol. 93, 582-584 (2021).

24. van Moorsel, C. et al. The Muc5B Promotor Polymorphism Associates With Severe Covid-19. preprint at https://doi.org/10.1101/2020.05.12.20099333 (2021).

25. Ho, J. C. et al. The effect of aging on nasal mucociliary clearance, beat frequency, and ultrastructure of respiratory cilia. Am. J. Respir. Crit. Care Med. 163, 983-988 (2001).

26. Biering, S. B. et al. Genome-wide, bidirectional CRISPR screens identify mucins as critical host factors modulating SARS-CoV-2 infection. preprint at https://doi.org/I0.I I 0I/202I.04.22.440848 (2021).

27. Plante, J. et al. Mucin 4 Protects Female Mice from Coronavirus Pathogenesis. preprint at https://doi.org/10.1101/2020.02.19.957118 (2020).

28. Bose, M., Mitra, B. \& Mukherjee, P. Mucin signature as a potential tool to predict susceptibility to COVID-19. Physiol. Rep. 9, 1-10 (2021).

29. Honigfort, D. J., Altman, M. O., Gagneux, P. \& Godula, K. Glycocalyx crowding with mucin mimetics strengthens binding of soluble and virus-associated lectins to host cell glycan receptors. Proc. Natl. Acad. Sci. 118, e2107896118 (2021).

30. Clausen, T. M. et al. SARS-CoV-2 Infection Depends on Cellular Heparan Sulfate and ACE2. Cell 183, 1043-1057.e15 (2020).

31. Nguyen L. et al. Sialic acid-Dependent Binding and Viral Entry of SARS-CoV-2. 10.21203/rs.3.rs$318912 / \mathrm{v} 1$.

32. Zhang, Q. et al. Heparan sulfate assists SARS-CoV-2 in cell entry and can be targeted by approved drugs in vitro. Cell Discov. 6, 80 (2020).

33. Liu, L. et al. Heparan Sulfate Proteoglycans as Attachment Factor for SARS-CoV-2. ACS Cent. Sci. 7, 1009-1018 (2021).

34. Chu, H. et al. Host and viral determinants for efficient SARS-CoV-2 infection of the human lung. 
Nat. Commun. 12, 1-15 (2021).

35. He, J. et al. Single-cell analysis reveals bronchoalveolar epithelial dysfunction in COVID-19 patients. Protein Cell 11, 680-687 (2020).

36. Nakashima, T. et al. Circulating KL-6/MUC1 as an independent predictor for disseminated intravascular coagulation in acute respiratory distress syndrome. J. Intern. Med. 263, 432-439 (2008).

37. Vestbo, J. Epidemiological studies in mucus hypersecretion. Novartis Found. Symp. 248, 3-12 (2002).

38. Guan, W. J., Chen, R. C. \& Zhong, N. S. Strategies for the prevention and management of coronavirus disease 2019. Eur. Respir. J. 55, (2020).

39. Kost-Alimova, M. et al. A High-Content Screen for Mucin-1-Reducing Compounds Identifies Fostamatinib as a Candidate for Rapid Repurposing for Acute Lung Injury. Cell Reports Med. 1, (2020).

40. Liu, Y. et al. Mucus production stimulated by IFN-AhR signaling triggers hypoxia of COVID-19. Cell Res. 30, 1078-1087 (2020).

41. Tabassum, N., Zhang, H. \& Stebbing, J. Repurposing Fostamatinib to Combat SARS-CoV-2Induced Acute Lung Injury. Cell Reports Med. 1, 100145 (2020).

42. Butler, A., Hoffman, P., Smibert, P., Papalexi, E. \& Satija, R. Integrating single-cell transcriptomic data across different conditions, technologies, and species. Nat. Biotechnol. 36, 411-420 (2018).

43. Whitt, M. A. Generation of VSV pseudotypes using recombinant $\Delta G$-VSV for studies on virus entry, identification of entry inhibitors, and immune responses to vaccines. J. Virol. Methods 169, 365-374 (2010).

44. Lamers, M. M. et al. Human airway cells prevent sars-cov-2 multibasic cleavage site cell culture adaptation. Elife 10, 1-22 (2021).

45. Li, X. et al. MUC1 is a receptor for the Salmonella SiiE adhesin that enables apical invasion into enterocytes. PLoS Pathog. 15, 1-20 (2019).

46. Widjaja, I. et al. Towards a solution to MERS: protective human monoclonal antibodies targeting different domains and functions of the MERS-coronavirus spike glycoprotein. Emerg. Microbes Infect. 8, 516-530 (2019). 
47. Schindelin, J. et al. Fiji - an Open platform for biological image analysis. Nat. Methods 9, (2009).

\section{Acknowledgements}

We thank Wentao Li and Arno van Vliet for assistance in virus production. We thank Robert P. de Vries (Faculty of Pharmaceutical Sciences, Utrecht University) for providing reagents for the heparan sulfate experiments. We thank Daphne Stapels for valuable input. MC is supported by One Health funding provided by the Faculty of Veterinary Medicine. KS is supported by the European Research Council under the European Union's Horizon 2020 research and innovation program (ERC-2019-STG-852452).

\section{Author Contributions}

MC and LZXH performed the majority of the experiments. CW and BJB provided materials and assisted with generation of pseudotyped SARS-CoV-2. BW performed analysis of single cell RNA-seq data. RWW assisted with confocal microscopy. AZM performed the authentic SARS-CoV-2 experiments under supervision of BLH. JPMP reviewed the manuscript. KS supervised and helped design all aspects of the study. MC and KS analyzed the results, created the figures, and wrote the manuscript. All authors reviewed the final version of the manuscript.

\section{Competing Interests}

We declare no conflict of interest.

\section{Materials and Correspondence}

Contact Karin Strijbis (K.Strijbis@uu.nl) for requests and correspondence. 


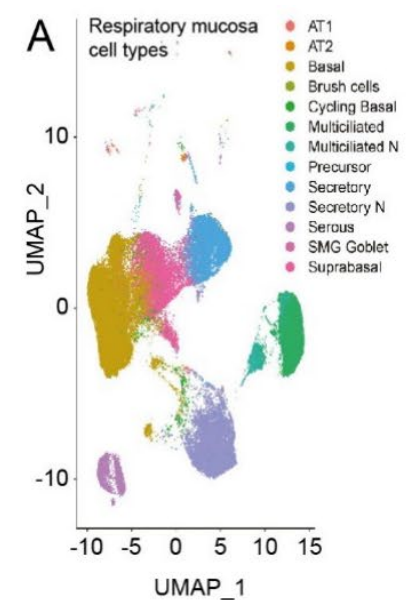

C

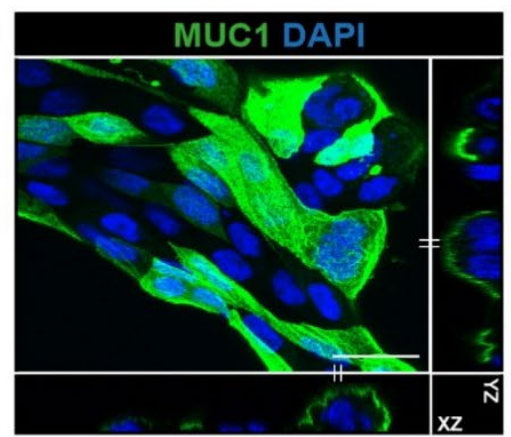

$\mathrm{D}$

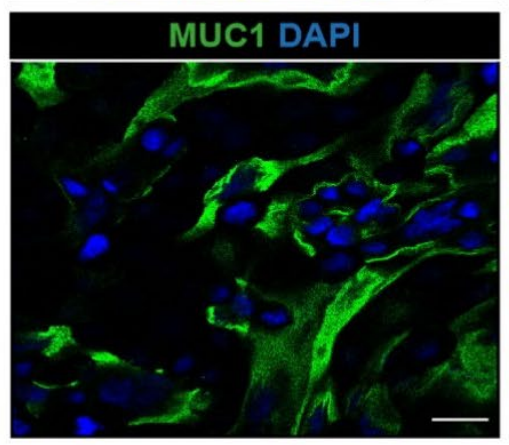

$\mathrm{E}$

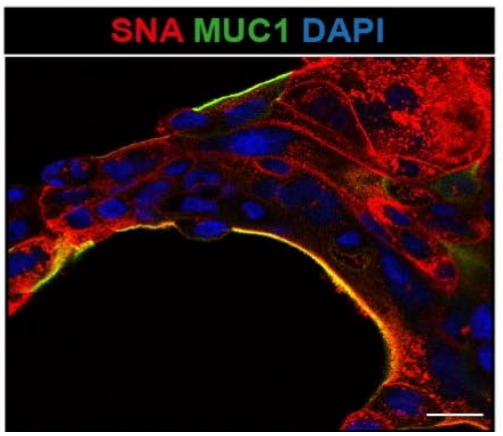

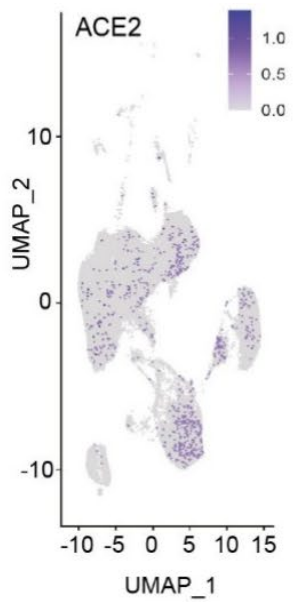

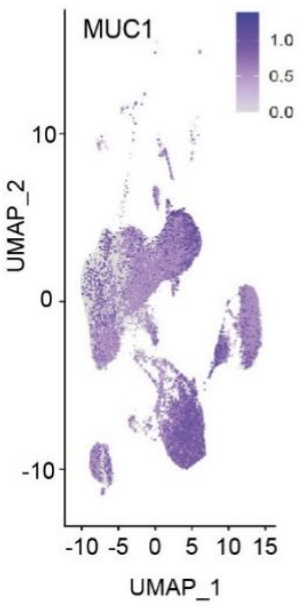

\section{B Expression in ACE2+ cells}
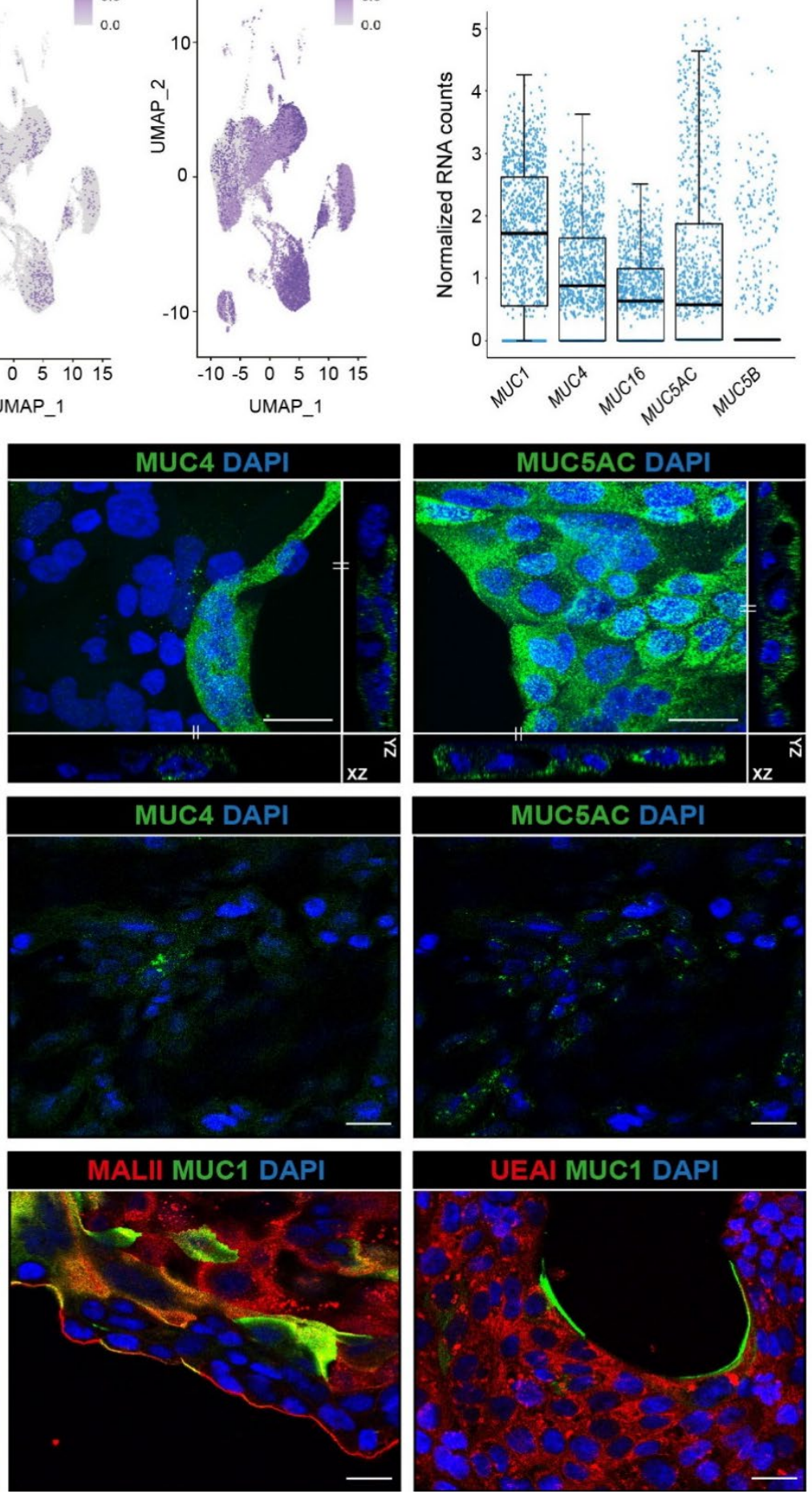

Figure 1. Expression of mucins in respiratory epithelial cells. (A) scRNA-seq analysis of ACE2 and MUC1 expression in different cell types in the respiratory mucosa. Dataset include samples from nasal cavity, upper, intermediate and lower respiratory tract ${ }^{15}$. (B) Expression of TM mucins MUC1, MUC4 and MUC16 and gel-forming mucins MUC5AC and MUC5B in ACE2 positive cells. MUC1 is the most highly expressed mucin in ACE2-positive cells. (C) Immunofluorescence confocal microscopy images showing expression of TM mucins MUC1 (214D4, green), MUC4 (8G7, green) and gel-forming mucin MUC5AC (MUC5AC, green) in Calu-3 cells. Maximum projections and side views of Z-stacks are shown. (D) Immunofluorescence confocal microscopy without permeabilization showing expression of MUC1 on 
the surface of Calu-3 cells. MUC4 and MUC5AC could barely be detected suggesting intracellular localization. (E) Immunofluorescence confocal microscopy imaging for $\alpha-2,6$ sialic acids (SNA, red), $\alpha$ 2,3 sialic acids (MALII, red) and fucose (UEA1, red) in combination with MUC1 (214D4 antibody, green) demonstrates high levels of sialic acid and fucose in Calu-3 cells. Nuclei were stained with DAPI (blue). White scale bars represent $20 \mu \mathrm{m}$. 

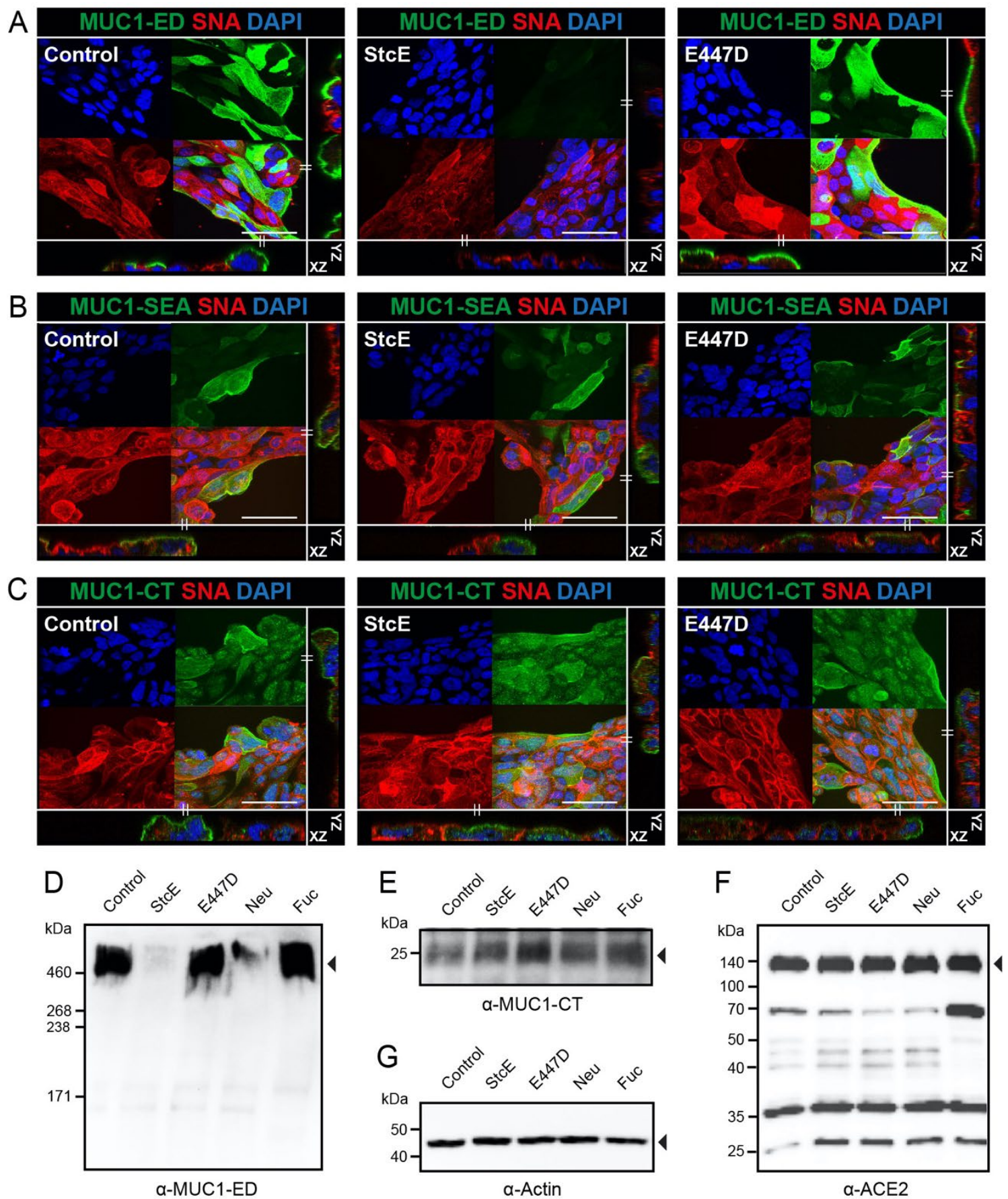

Figure 2. StcE specifically cleaves the glycosylated MUC1 ED and does not affect ACE2 expression.

(A) Immunofluorescence confocal microscopy images showing Calu-3 cells treated with StcE or E447D stained for the glycosylated part of the MUC1 extracellular domain (214D4, green) and $\alpha$-2,6-linked sialic acids (SNA, red). Complete loss of 214D4 signal was observed after treatment with StcE. (B,C) Immunofluorescence confocal microscopy images of Calu-3 cells as above stained for the MUC1 SEA domain ( $\alpha$-MUC1-SEA antibody 232A1, green) or cytoplasmic tail of MUC1 ( $\alpha$-MUC1-CT antibody CT2, green) in combination with $\alpha-2,6$-linked sialic acids (SNA, red). The SEA domain and CT were not affected by StcE treatment. Nuclei were stained with DAPI (blue). White scale bars represent $20 \mu \mathrm{m}$. Western blot analysis of 7-day grown Calu-3 cells incubated with indicated enzymes for $3 \mathrm{~h}$ at $37^{\circ} \mathrm{C}$ stained with $\alpha$-MUC1-ED antibody 214D4 (D), the MUC1 cytoplasmic tail with $\alpha$-MUC1-CT antibody CT2 (E), ACE2 (F) and $\beta$-actin loading control (G). StcE treatment removes the MUC1 ED but does not affect the MUC1 CT or ACE2 receptor. 

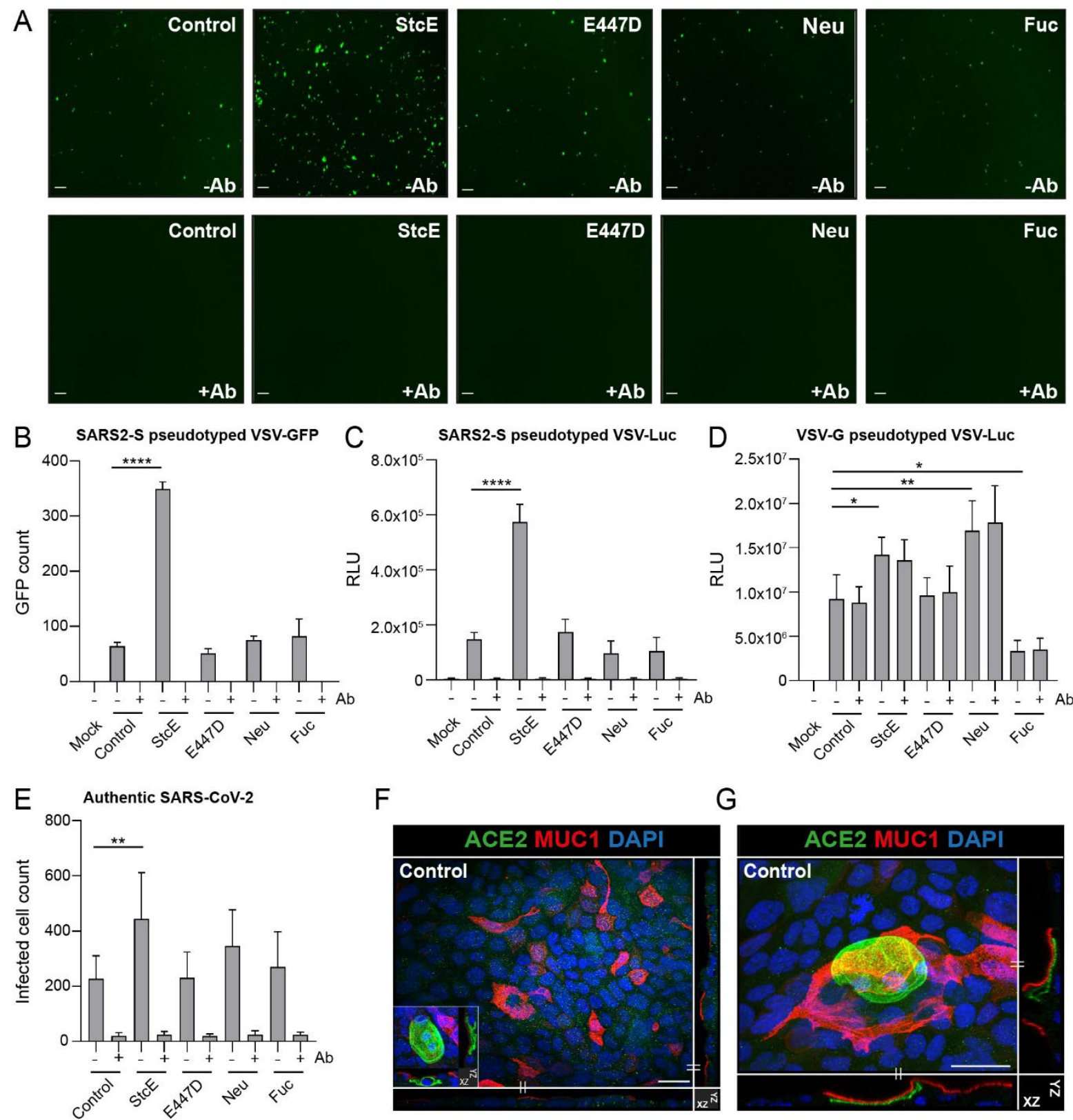

G
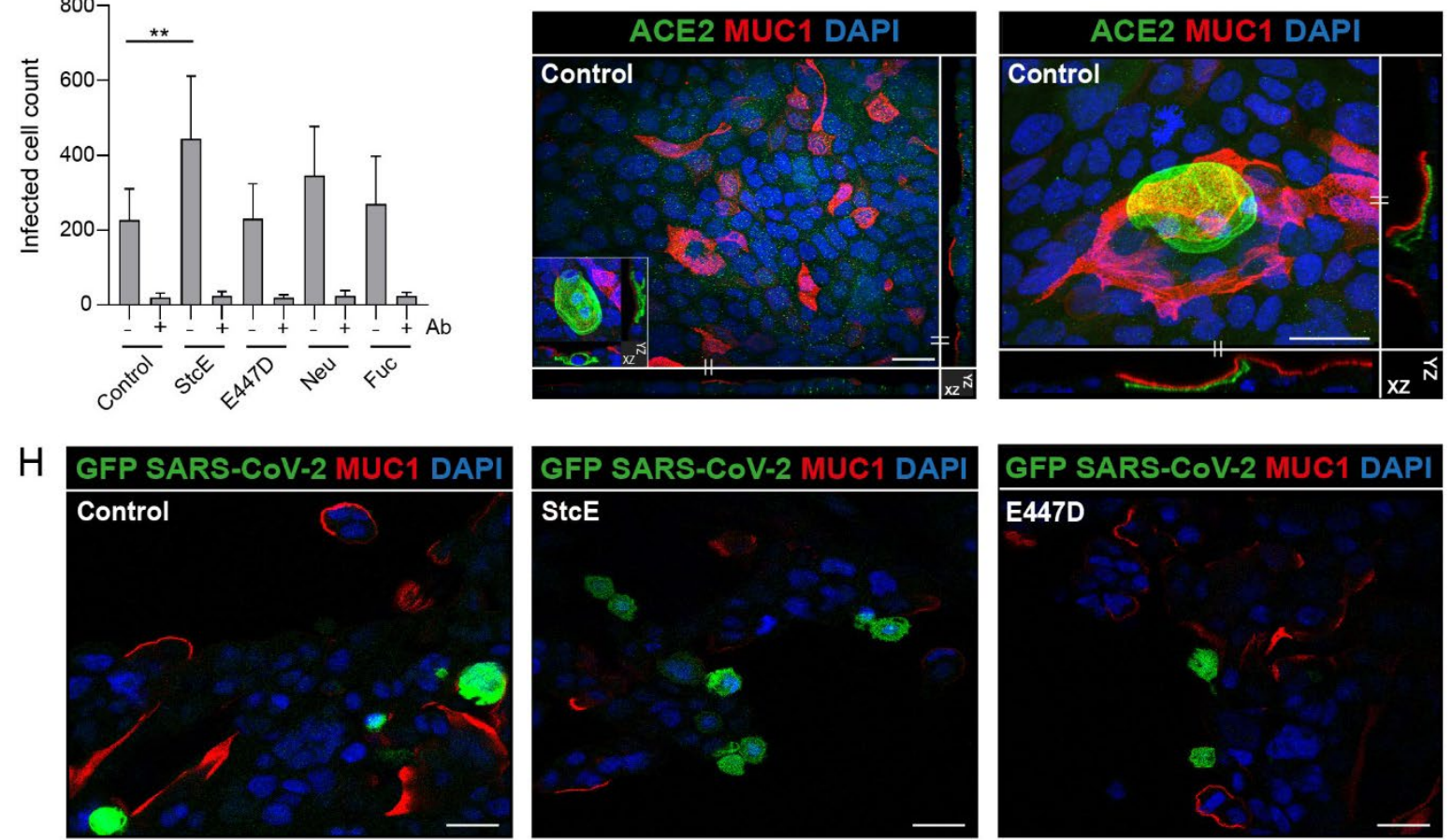

Figure 3. Removal of the glycosylated MUC1 extracellular domain enhances SARS-CoV-2 entry.

(A) Microscopy images of Calu-3 cells treated with StcE, E447D, neuraminidase or fucosidase infected with SARS2-S pseudotyped VSV-GFP without or with neutralizing monoclonal antibody (mAb) against SARS2-Spike. White scale bars represent $200 \mu \mathrm{m}$. (B) Quantification of SARS2-S pseudotyped VSV-GFP signal in Calu-3 cells using EVOS software. StcE treatment resulted in a 5.4-fold increase in infection. 
(C) Quantification of luciferase signal (RLU) in Calu-3 cells after treatment with indicated enzymes and infection with SARS2-S pseudotyped VSV-Luc in the absence or presence of mAb against spike. A 4fold increase in RLU value was observed when cells were treated with StcE. (D) Quantification of Calu3 cell infection with VSV-G pseudotyped VSV-Luc lacking the spike protein. Infection was not blocked by the anti- spike mAb. (E) Infection of Calu-3 cells with authentic SARS-CoV-2 after treatment with indicated enzymes. StcE treatment resulted in a 2 -fold increase in infected cell count. Neuraminidase and fucosidase treatment did not significantly impact viral entry. Represented values are the mean \pm SEM of three biological replicates performed in triplicate. Statistical analysis was performed by repeated measures one way-ANOVA with Dunnett's post-hoc test. $p>0.05$ [ns, not significant], $p<0.05$ $\left[{ }^{*}\right], p<0.01\left[{ }^{* *}\right], p<0.001\left[{ }^{* * *}\right], p<0.0001[* * * *] .(F, G)$ Immunofluorescence confocal microscopy for localization and distribution of ACE2 (green) and TM mucin MUC1 (214D4, red) in Calu-3 cells. For ACE2, a general punctated pattern was observed with the occasional appearance of dome-like structures that were overlayed by MUC1. (H) Immunofluorescence confocal microscopy imaging of Calu-3 cells treated with mucinase and incubated with SARS2-S pseudotyped VSV-GFP (green) for 18$20 \mathrm{~h}$ stained with anti-MUC1 214D4 antibody (red) and DAPI (blue). White scale bars represent $20 \mu \mathrm{m}$. 
A
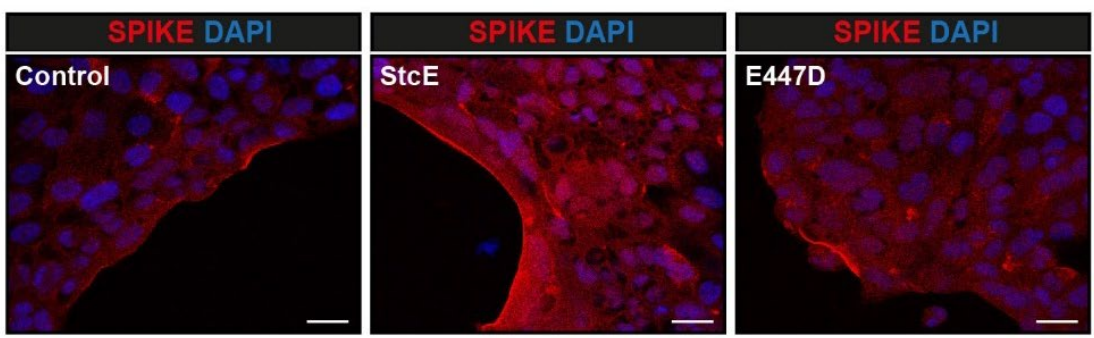

B
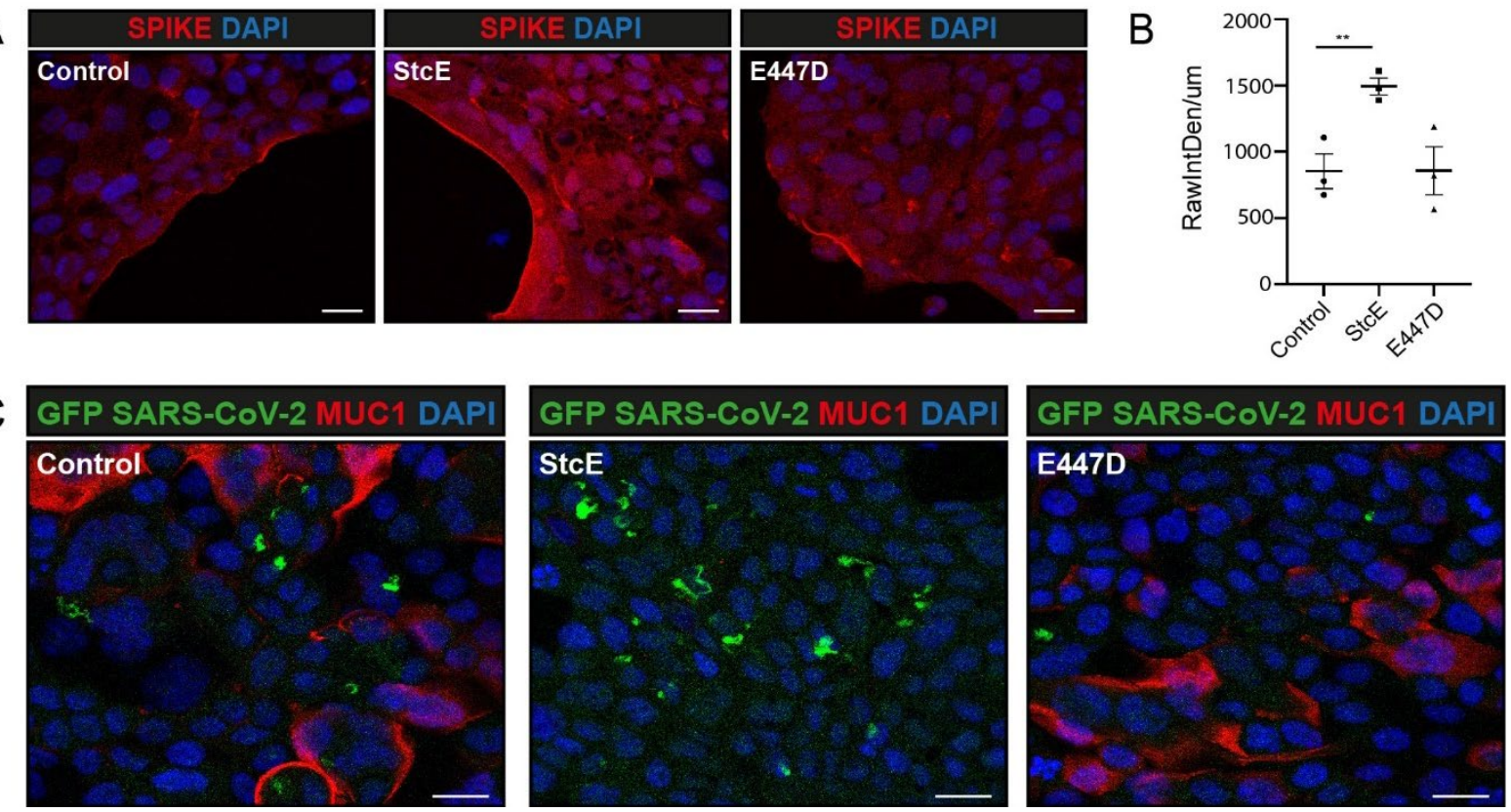

Figure 4. Removal of the MUC1 extracellular domain increases spike and virus attachment.

(A) Immunofluorescence confocal microscopy of Calu-3 cells incubated with $2.5 \mathrm{ug} / \mathrm{ml} \mathrm{SARS-CoV-2}$ spike (Fc-tagged SARS2-S1B-Fc, red) at $4^{\circ} \mathrm{C}$ for $1 \mathrm{~h}$. Spike was stained without permeabilization. Increased spike binding and higher spike signal intensity was observed after treatment with StcE in comparison to E447D treatment and control. (B) Quantification of spike fluorescence signal as depicted in A. Fluorescence intensity along the edge of cell island was determined in control, StcE- and E447D-treated cells using ImageJ. Mean \pm SEM raw integrated density/length from three random fields from three independent experiments are plotted. The area of spike binding was significantly higher in StcE-treated cells. (D) Immunofluorescence confocal microscopy of Calu-3 cells incubated with SARS2-S pseudotyped VSV-GFP (green) at $4^{\circ} \mathrm{C}$ for $1 \mathrm{~h}$. Complete disappearance of MUC1 ED signal (214D4, red) and increased virus attachment (green) and was observed in StcE-treated cells. Nuclei were stained with DAPI (blue). White scale bars represent $20 \mu \mathrm{m}$. 


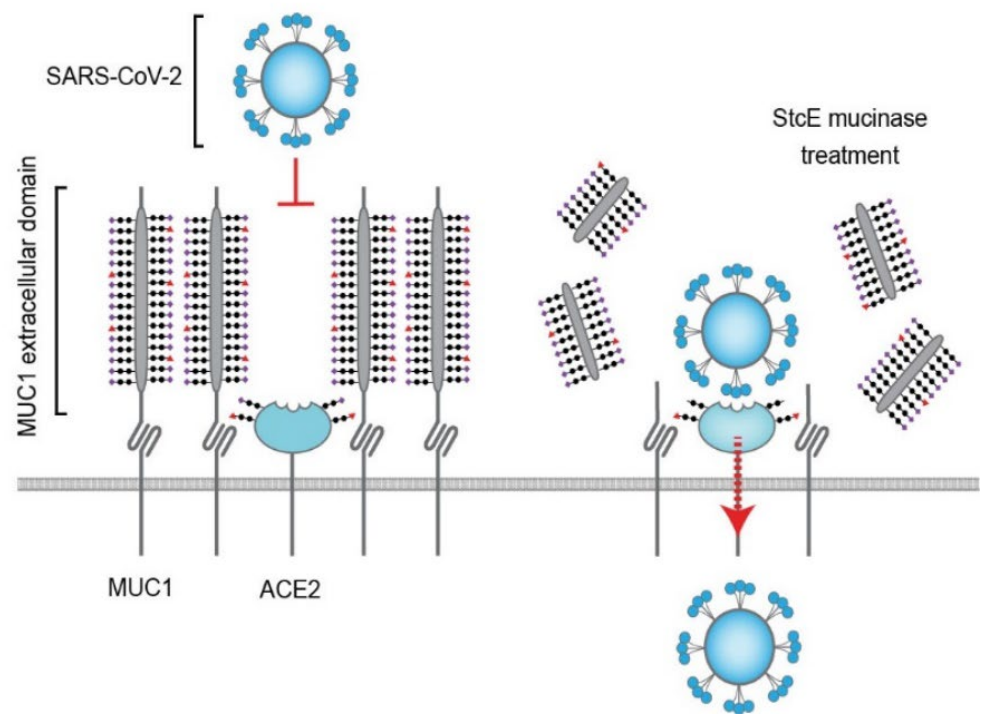

Figure 5. MUC1 protects against SARS-CoV-2 infection at the respiratory surface.

Working model describing the protective function of the MUC1 extracellular domain during SARS-CoV2 infection. The glycosylated extracellular domain of MUC1 prevents access of the virus to the ACE2 receptor (left). Enzymatic removal of the MUC1 extracellular domain with the StcE mucinase allows the viral spike protein to connect with the ACE2 receptor resulting in viral entry into lung epithelial cells (right). 